\title{
The impact of non-medical interventions on temporal behaviour of COVID-19 pandemic
}

\author{
Arindam Kushagra \\ Amity Institute of Nanotechnology, Amity University Kolkata, INDIA- 700156. \\ email: arindam.kushagra@gmail.com
}

\begin{abstract}
The ongoing pandemic, COVID-19, caused by the spread of respiratory SARS-CoV virus has brought the entire world to a halt. All the economic activities have greatly suffered because of this communicable disease. Governments all across the globe have been devising measures to control the spread of the ongoing epidemic by trying to keep a check on the number of daily infected cases and daily deaths and trying to minimize them by putting in tremendous amount of efforts. In this article, an analysis about the impacts of various governmental steps has been made in order to alter the temporal behaviour of the ongoing epidemic. The data considered for this work was till $17^{\text {th }}$ April, 2020. This work would be of interest to the policy-makers, health professionals, drug manufacturing companies \& to the common public alike and would be helpful in the formulation of subsequent mitigation strategies to fight this disease.
\end{abstract}

\section{Introduction}

COVID-19 started to spread from Wuhan district in Hubei province of China. It is caused due to acute respiratory distress following the infection by SARS-CoV-2. The spread of the virus happens by its absorption through soft tissues like mouth, eyes $\&$ nose. The disease spread very fast across the globe with high infectivity, given by the reproduction number $\mathrm{R}_{\mathrm{o}}(\sim 3)$ [1]. To contain its spread and mitigate the fatalities caused due to COVID-19, governments worldwide have been engrossed in dousing the wildfire-like situation due to the similar spread of this disease. Social-distancing has been accepted to be the norm unless the spread of the virus flatlines $[2,3]$. There have been predictions and reports that due to such socialdistancing measures, the numbers of infected $\&$ deceased were contained to a great extent. In this work, country-wise temporal behaviour of the COVID-19 pandemic has been discussed 
31 and to what extent the stringencies imposed by the respective governments affected the 32 flattening of the mortality curve. This work would further be of interest to policy-makers, 33 health professionals and other individuals who are involved in the mitigation of the effects 34 caused due to COVID-19.

36 Temporal behaviour of COVID-19: The temporal behaviour of the disease was studied by 37 plotting the number of cases reported daily against the number of days elapsed since the first 38 case was reported [4]. The first case was considered when there were non-zero reported cases 39 for two consecutive forthcoming days. The plotted data was fitted using a polynomial of 40 order three (03). Such studies were conducted for the top affected countries across the globe, 41 viz, Belgium, China, France, Germany, Iran, Italy, South Korea, Spain, Switzerland, United 42 Kingdom and United States, respectively [5]. The remaining countries which were not there 43 in the list were picked up on the basis that the fitted-curve should have passed the maximum 44 point and should have started moving downwards. A representative case of Iran is shown in 45 Fig. 1, as follows. 


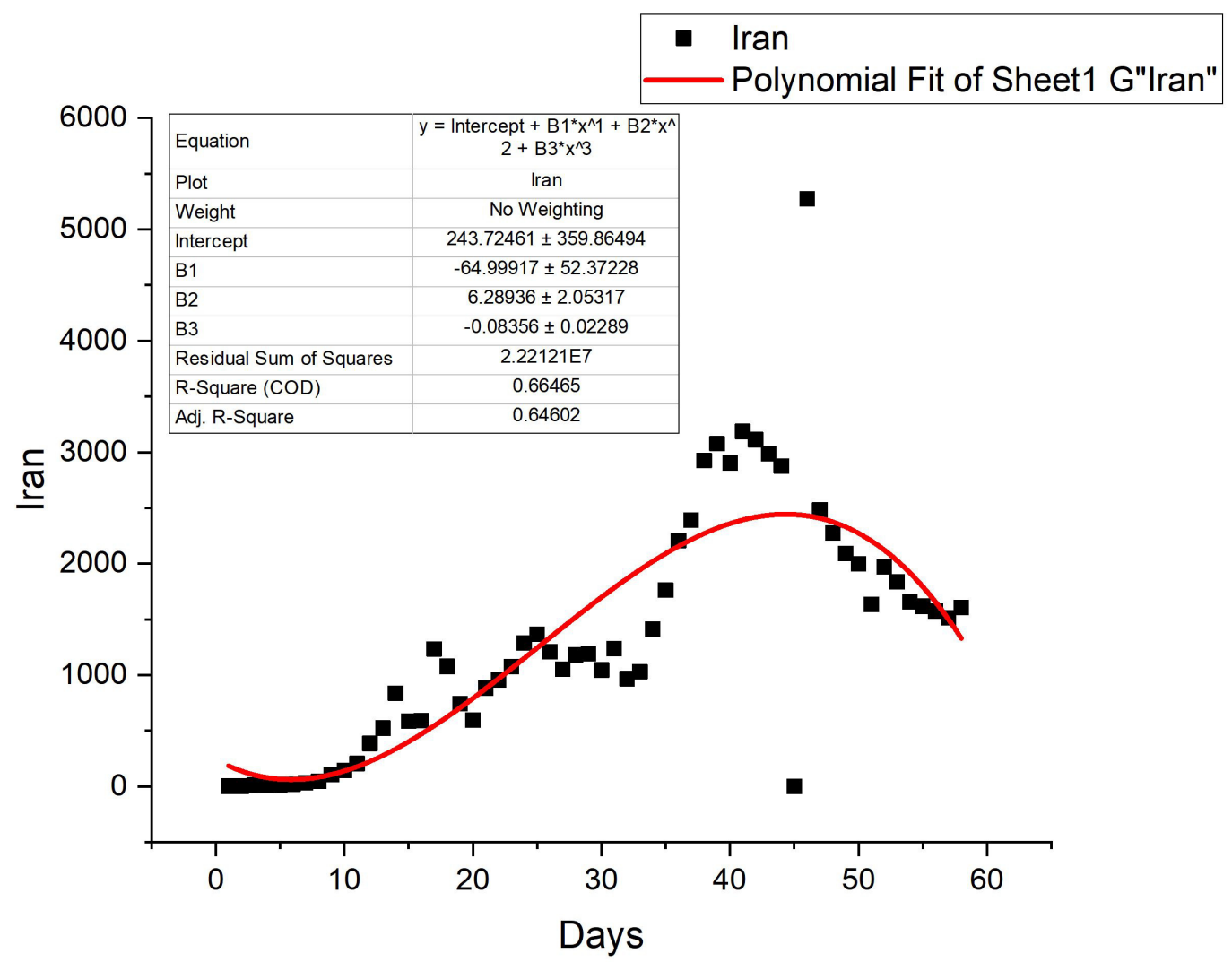

47 Fig. 1 Daily number of reported COVID-19 cases for Iran has been shown. As the red fitting 48 curve indicates, the cases in the country have started to decline and the pandemic is past its 49 peak.

50 A comprehensive treatise regarding the analysis that has been presented in Fig. 1 is given in 51 Section-A of the supplementary information. From the fitted curves as shown, it can be 52 deduced that the median peak-time (i.e. minimum-to-maximum daily cases) for the reported 53 countries is 40 days.

54 Dynamic governmental stringencies in response to COVID-19: Blavatnik School of 55 Government, University of Oxford, has devised a metric system to quantify the stringent 56 measures imposed by governments of respective countries in response to the dynamic 57 behaviour of COVID-19 [6]. Such governmental measures seem to be well-in-sync with the 58 temporal behaviour of the disease in that country. A representative case of Iran is shown in 59 Fig. 2. 


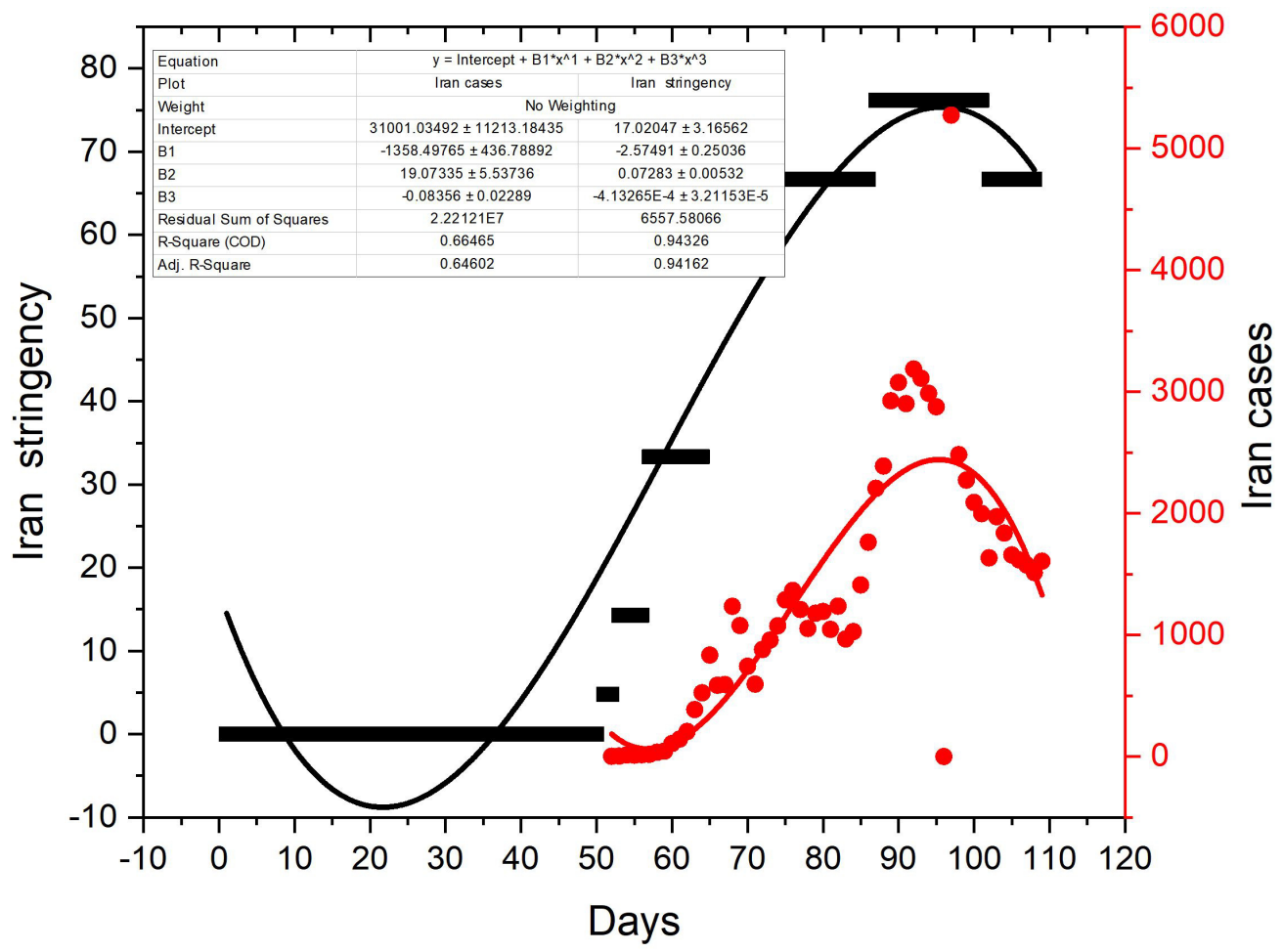

60

61 Fig. 2 Synchronous stringent measures by Iranian government with the progression of 62 COVID-19, is shown.

63 A comprehensive treatise of stringent measures of all the governments with dynamic 64 behaviour of COVID-19 is shown in Section-B of the supplementary information. The fitted 65 black and red curves indicate that the response from the respective governments were well66 timed and synchronous.

68 From the analyses done using the available data [4, 6], we obtain the following pattern of 69 country-wise peak-time behaviour and the maximum values of scaled stringencies as shown 70 in Fig. 3. 


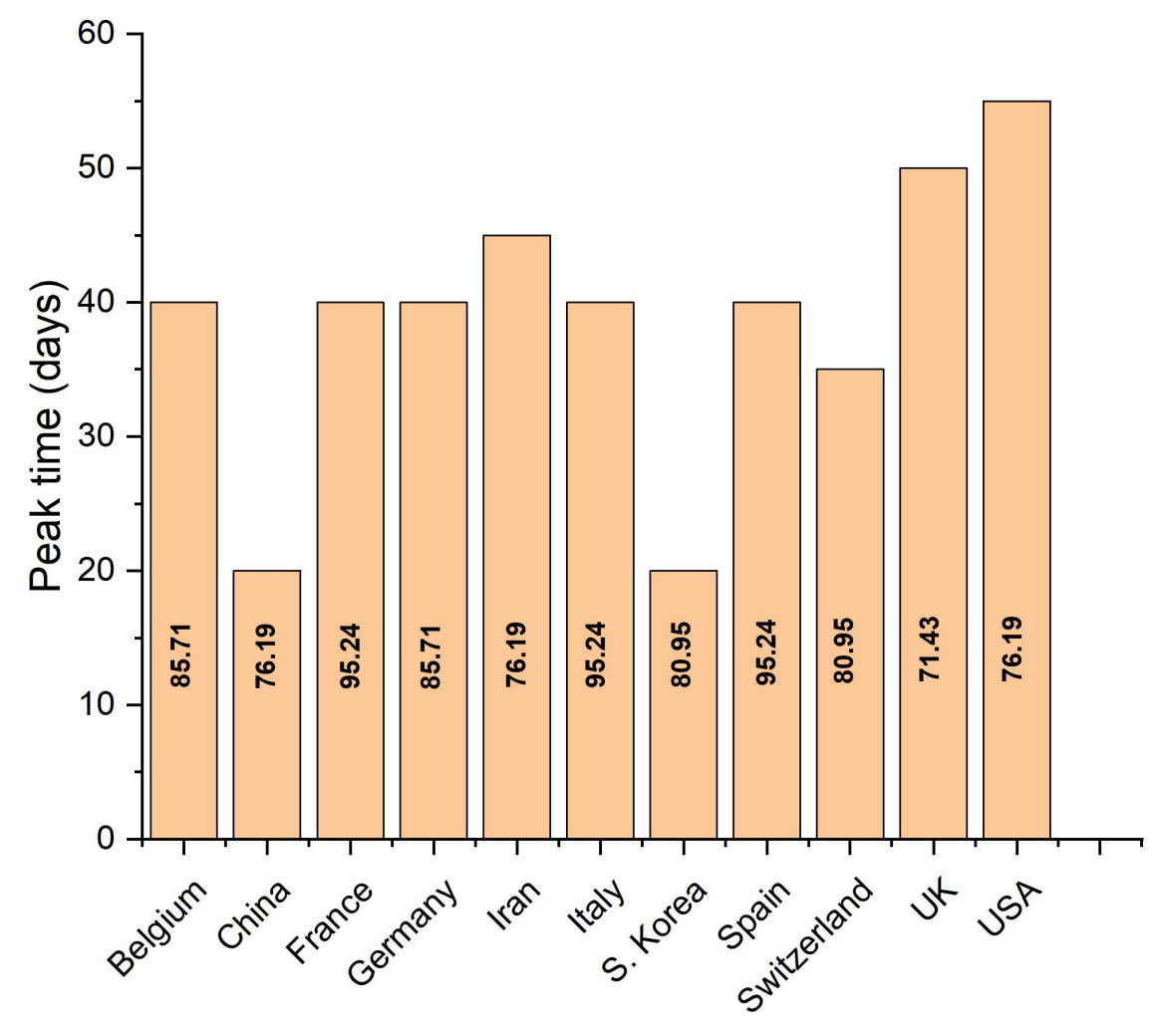

72 Fig. 3 Bar-chart showing the approximate peak time values for different countries with the maximum values of scaled stringencies imposed by the respective governments (inscribed within individual bars).

From Fig. 3 it is evident that despite having the stringency scores of 76.19 and 80.95, both China as well as South Korea got the peaks in approximately 20 days whereas the scores of $85.71 \& 95.24$ has the peak time value of approximately 40 days (for Germany, France, Italy \& Spain). Iran and U.S.A. despite having same stringency scores (76.19) have different peak times of approximately 45 days and 55 days, respectively. Thus, there does not seem a direct correlation of the peak time values with governmental stringency scores given by University of Oxford \& such scores cannot be conclusively used to determine the containment of the disease, as determined by the peak time values and flatlining of the mortality curves.

\section{Conclusions}

84 COVID-19 has secured the attention of modelers [7, 8] and financial analysts [9] to predict its 85 course that would help the entire world to get back on track, as fast as possible. In this article, 
the temporal behaviour of COVID-19 pandemic has been discussed and it was observed that the median peak time value is 40 days (for a data set of 11 most affected countries [5]). This result seems to be in accordance with the predictions made in reference [3]. Thus if the upswing and downswing of the time-dependent behaviour of the disease is assumed to be symmetrical, it can be concluded that the disease would automatically flatline after approximately 80 days. To mitigate the effects of this pandemic, different governments have been trying to impose some restrictions on its citizens so that the spread of the disease could be contained. These moves though shown to quantitatively suppress the numbers of mortalities and further spread of the disease seem to have no correlation with the temporal behaviour of the disease. This work would be of interest to researchers and scientists working in the field of public policy making, healthcare industries, drug manufacturing companies and to the social scientists to formulate relevant strategies to sail through these difficult pandemic times.

\section{Conflict of interests}

The author declares no conflict of interest.

\section{Author contributions}

A.K. formulated the problem statement, did the analysis and wrote the manuscript.

\section{References}

[1].Y. Liu, A.A. Gayle, A. Wilder-Smith, J. Rocklov, The reproductive number of COVID-19 is higher compared to SARS coronavirus, J Travel Med. 2020 Mar $13 ; 27(2)$.

[2].M.U.G. Kramer et al., The effect of human mobility and control measures on the COVID-19 epidemic in China, Science. 2020 Mar 25. pii: eabb4218.

[3].R. Singh, R. Adhikari, Age-structured impact of social distancing on the COVID-19 epidemic in India, arXiv:2003.12055 [q-bio.PE].

[4].Coronavirus Disease (COVID-19) - the data, https://ourworldindata.org/coronavirusdata, accessed on $19^{\text {th }}$ April, 2020. 
113 [5].How coronavirus is spreading around the globe - an infographic, 114 https://www.weforum.org/agenda/2020/03/infographic-coronavirus, accessed on $19^{\text {th }}$ $115 \quad$ April, 2020. https://www.bsg.ox.ac.uk/research/research-projects/coronavirus-governmentresponse-tracker, accessed on $19^{\text {th }}$ April, 2020.

[7].D. Adam, Special report: The simulations driving the world's response to COVID-19, https://www.nature.com/articles/d41586-020-01003-6, accessed on 19 ${ }^{\text {th }}$ April, 2020.

[8].J.R. Gog, How you can help with COVID-19 modelling, Nat Rev Phys. 2020. https://doi.org/10.1038/s42254-020-0175-7

[9].W. Cornwall, Can you put a price on COVID-19 options? Experts weigh lives versus economics, https://www.sciencemag.org/news/2020/03/modelers-weigh-value-livesand-lockdown-costs-put-price-covid-19\#, accessed on $19^{\text {th }}$ April, 2020. 
Supplementary Information for

The impact of non-medical interventions on temporal behaviour of COVID-19

$$
\text { pandemic }
$$

$$
\text { by }
$$

Arindam Kushagra

Amity Institute of Nanotechnology, Amity University Kolkata, INDIA- 700156. email: arindam.kushagra@gmail.com 


\section{Section -A}

Determination of country-wise peak-time values [1]
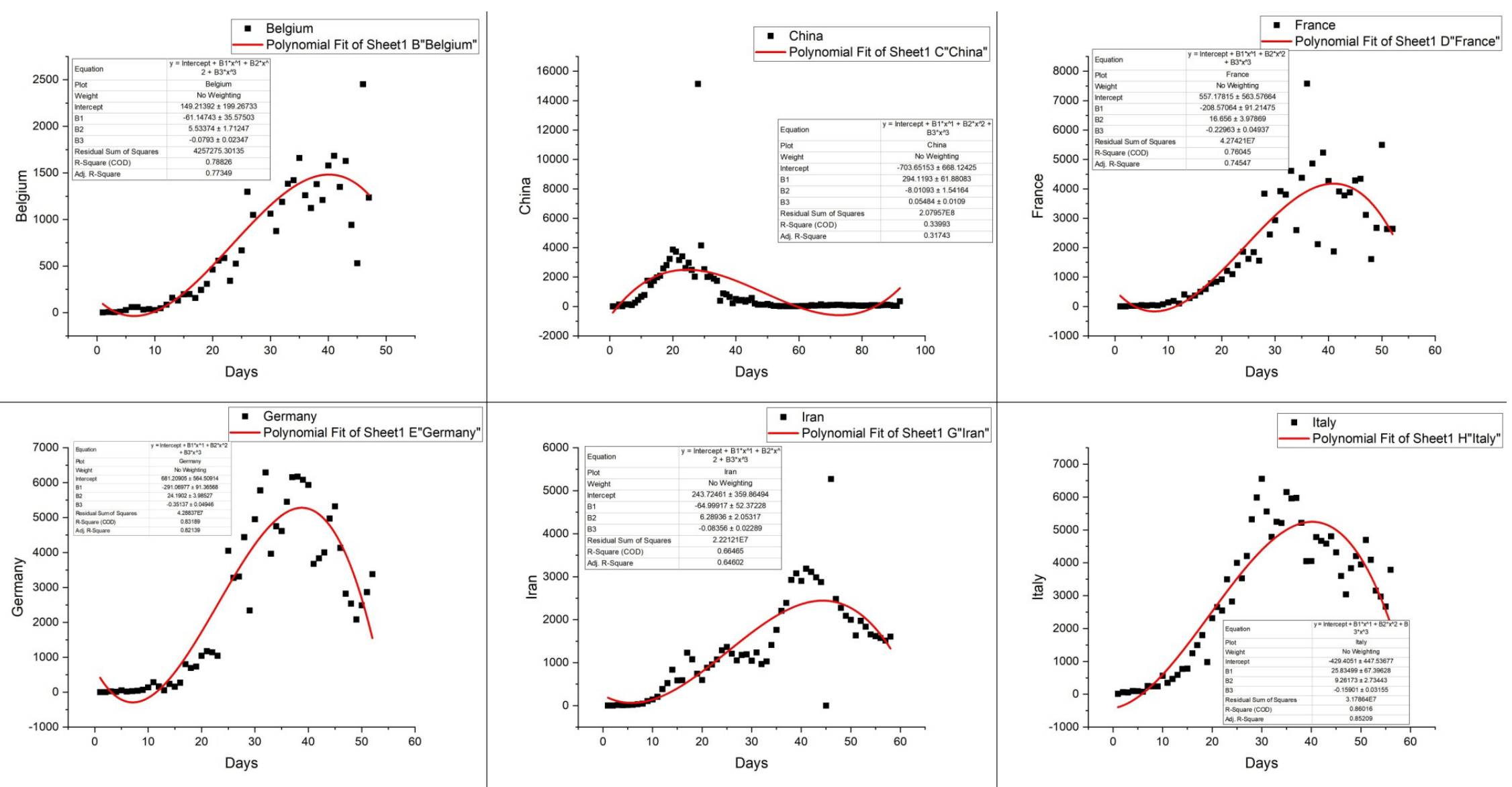

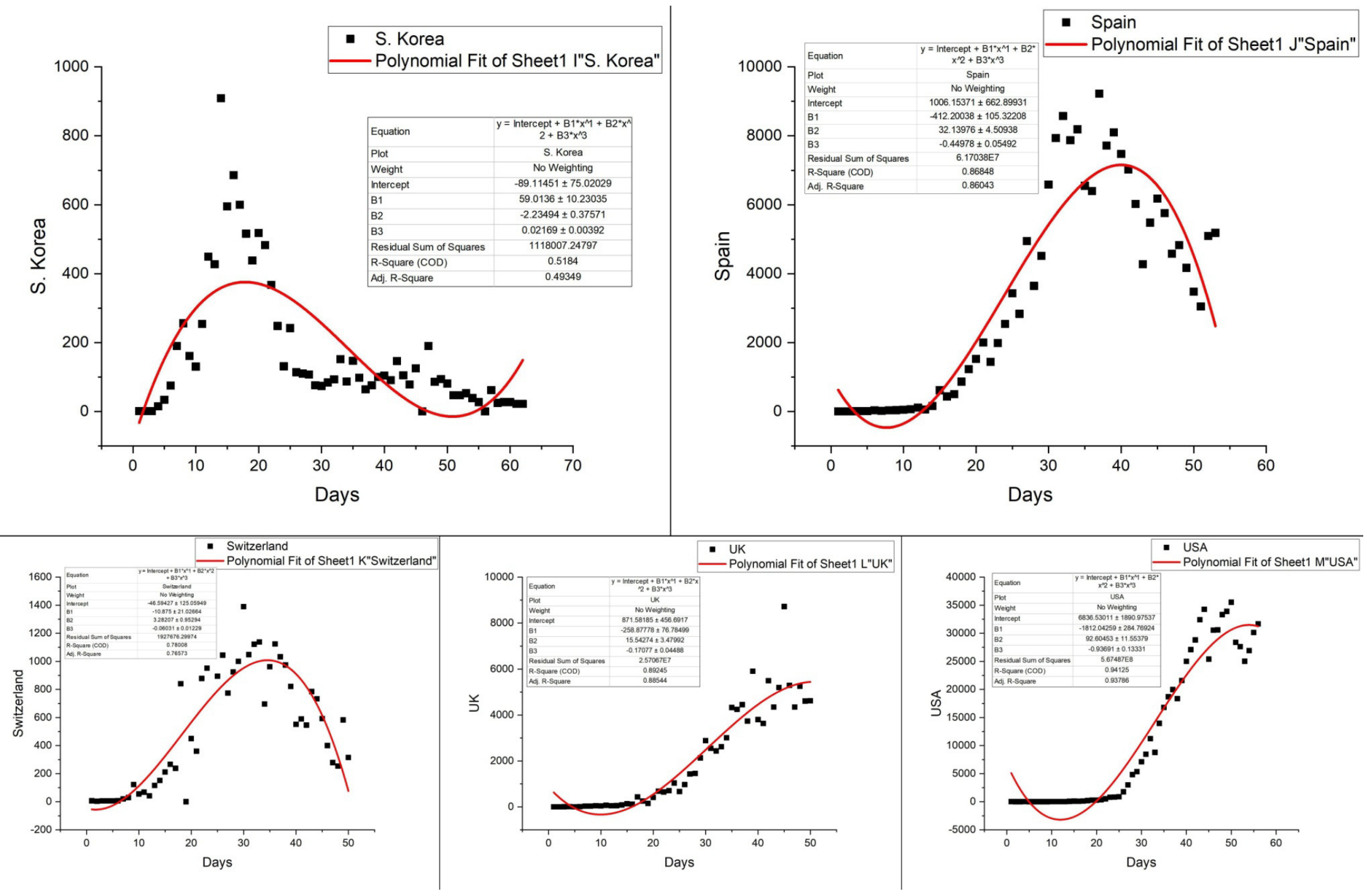


\section{Section -B}

Time-dependent governmental stringencies in response to COVID-19 pandemic [1, 2]

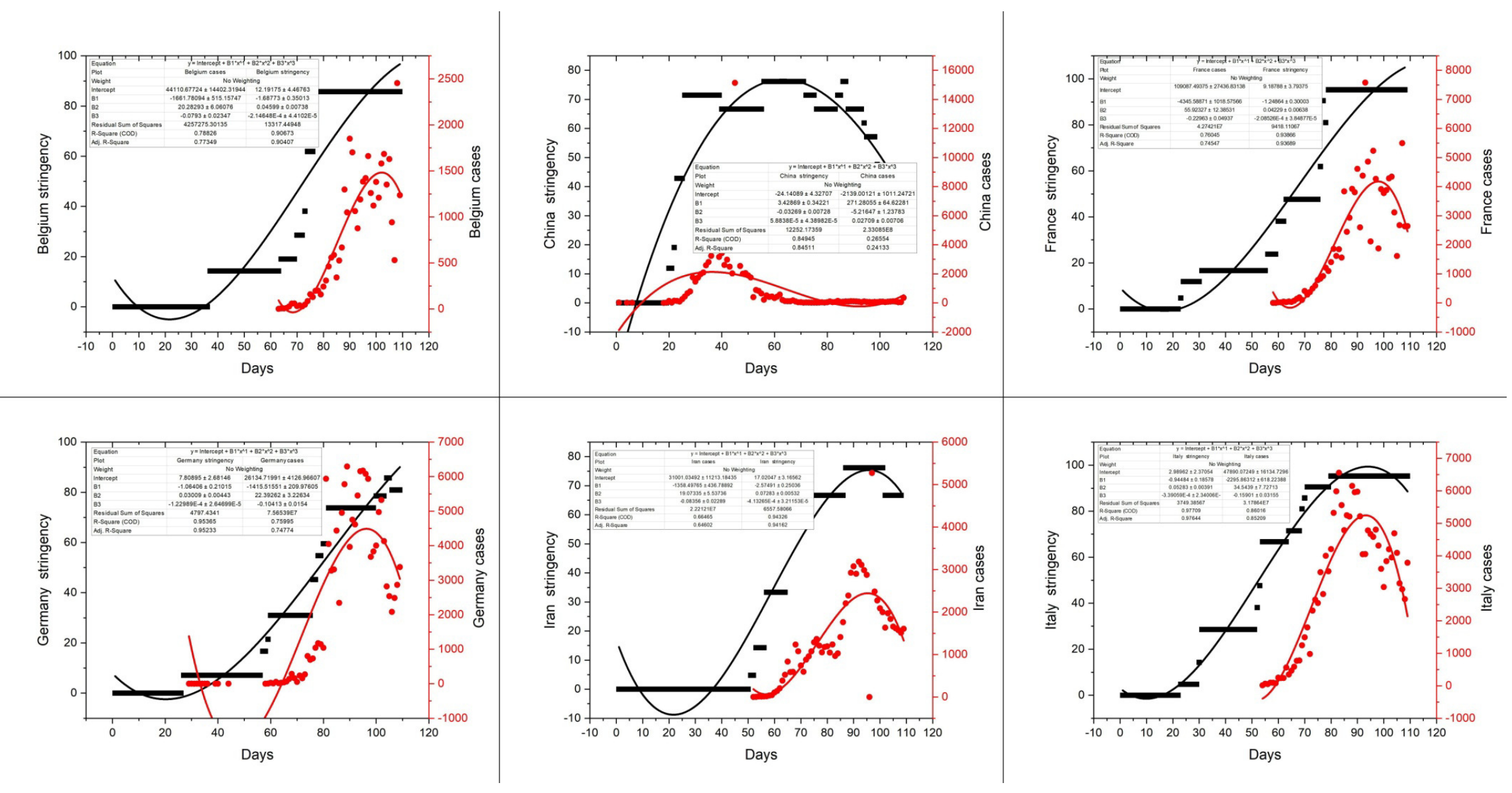



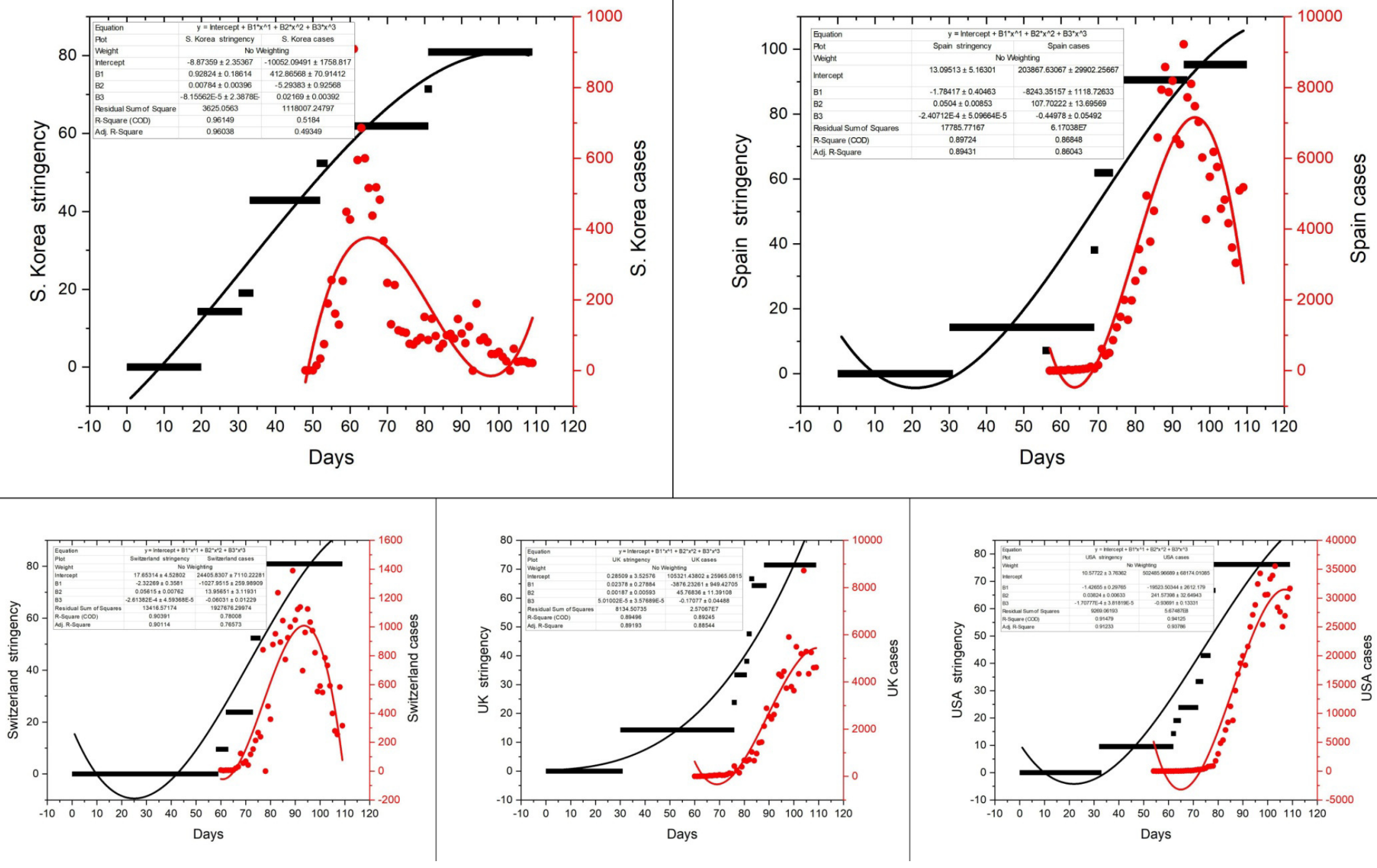


\section{References}

[1]. Coronavirus Disease (COVID-19) - the data, https://ourworldindata.org/coronavirus-data, accessed on 19 ${ }^{\text {th }}$ April, 2020.

[2].Coronavirus Government Response Tracker, https://www.bsg.ox.ac.uk/research/research-projects/coronavirus-government-responsetracker, accessed on $19^{\text {th }}$ April, 2020. 